DEFENSIVOS QUTMICOS (ESPECIALMENTE NOVOS PRODUTOS) NO COMBATE AO ACARO RAJPDO Tetranychus urticae $\mathrm{KOCH}$, 1836, EM CULTURA DE ALGODAO*
A.J. RAIZER*
J.M. SILVA**
M. TAKAOKA**
R. MOTTA**
W. Y. KATOK*
F.A.M. MARICONI***

Rli:iUM:

Este experimento vem dar continuidade às pesquisas de combate ao ácaro rajado do algodoei ro T'etranzuhus urtivit' Koch, 1836, tendo por objetivo prinicipal encontrar produtos eficientes contra esse aracnídeo.

Nos sete tratamentos realizados no campo, incluída a testemunha, foram utilizados quatro produtos: b ifentrina $(50 \mathrm{e}$

* Entregue para publicaçāo em 20/12/85.

** Bolsistas do Departamento de Zoologia, E.S.A. "Luiz de Cueiroz", USP

*** Departamento de Zoologia, E.S.A. "Luiz de Queiroz", USP. 
$\left.7^{n} \mathrm{~g} / \mathrm{ha}\right)$, bromopropilato $(500 \mathrm{~g} / \mathrm{ha})$, car bamato UC 81341 (100 e $150 \mathrm{~g} / \mathrm{ha})$ e cloF fent zina $(500 \mathrm{~g} / \mathrm{ha})$.

A żnālise dos resultados demostrou que a clofentezina foi o único tratamento eficaz, pois apresentou ótimos resultados contra o ácaro e um efeito residual relativamente longo.

Palavras-chave: Tetranychus urticae, de fensivos químicos, àcaro rajado, algodoeiro.

I NTROOUÇÃO

o presente trabalho vem dar prosseguimento a uma série de experimentos que visam o combate químico do àca ro T'etranychus urticae Koch, 1836, realizados pelo Depar partamento de Zoologia.

Esse aracnídeo, vulgarmente conhecido como ácaro rajado do algodoeiro, é possivelmente, ainda a principal praga dessa cultura na região de Piracicaba, Estado de São Paulo, atingindo máximo grau de infestação nos meses de janeiro e fevereiro.

As pesquisas no combate ao ácaro rajado do algodoeiro vêm se intensificando; são de elevada importância devido a dificuldade existente no seu controle. Este àca ro apresenta elevada resistência aos inseticidas e acar $\bar{i}$ cidas, podendo prejudicar seriamente a cultura de algodão se no campo encontrar condições favoräveis para sua proliferação. Na região de Jaboticabal, Estado de São Paulo, OLIVEIRA (1971) pesquisou os efeitos dos diversos niveis de infestação do ácaro no algodoeiro, consta 
tando perda de 7,8 a $25,5 \%$ na produção, expressa em peso de algodão em caroço, havendo prejuízos significativos na qualidade da fibra e semente.

Em busca de um combate eficiente dessa praga, diversos autores realizaram experimentos com diferentes produtos químicos e dosagens de produto ativo. Dentre eles, resultam os de PEREZ et alii (1978) que, pulverizando diferentes produtos, verificam que as pulverizaçōes realizadas de baixo para cima são mais eficientes que as normais. MORAls et alii (1981) testam eficiència de inseticidas no combate ao "äcaro rajado" em algodoeiro, obtendo resultados promissores em duas aplicaçōes utilizando UC-55248 e amitraz. MARICONI et alii (1983) realizam experimento em Santa Bärbara D'0este, SP, verificando que os piretróides FMC 54800 (bifentrina) e FMC 54617, e ainda a mistura profenofös + bromopro pilato conduziram a bons resultados aos 4 dias após a aplicação. SANTOS et alii (1983) montam campo experimen tal na região de Piracicaba, e a interpretação dos dados referentes ao combate químico ao aracnídeo não revelou nenhum produto promissor. ALMEIDA et alii (1984) aplicam defensivos quimicos em algodão e verificam que a clo fentezina mostra-se promissora no combate ao ácaro rajado. Em Assaí, Estado do Paranä, ALVES \& SALGADO (1984) constatam que o produto MK $9361,8 \%$ EC na dose de $10 \mathrm{~g}$ $\mathrm{A} / \mathrm{ha}$ apresenta eficiēncia superior aos padróes.

KATO et alii (1984) obtēm resultados relativos com a fempropa trina em pulverização contra o ácaro. PEDRONI et ali (1984) verificam a dificuldade intensa de combate ao aracnídeo, tanto com produtos Electrodyn, como com convencionais. SANTOS (1984) realiza ensaio na região de Ibiporã, Estado do Paraná, verificando que a avermectina na dose de 7,2 g lA/ha mostrou-se eficiente durante 55 dias de avaliação. TAKAOKA et alii (1984) testam dife rentes produtos, observando que o carbamato UC 81341 , nas doses de 150 e $300 \mathrm{~g}$ de IA por hectare, mostrou-se promissor.

O objetivo deste trabalho e subsidiar os estudos de combate desta importante praga do algodoeiro. 
MATERIAIS E METODOS

Local

Campo experimental instalado em propriedade do $\mathrm{Sr}$. Newton Mac Knight, localizada em Santa Bärbara D'Ces te, SP.

\section{Campo Experimental}

Formado de algodão variedade $I A C-17$, plantados em: espaçamento de $0,95 \mathrm{~m}$.

\section{Tratamentos}

Em nümero de sete, com quatro repetiçōes, num total de 28 parcelas de $200 \mathrm{~m}^{2}$, dispostas em blocos casuali zados. Os tratamentos, formulações, concentrações e con sumos de material se encontram na Tabela 1.

\section{Aplicaçào}

Realizada em 13/01/85, com dois atomizadores costais "Jacto", com capacidade para 12 litros; entretanto, somente a quantidade de calda necessāria para uma parcela era colocada em cada aparelho e pulverizada; em cada uma das outras parcelas colocava-se igual quantidade; des sa forma cada tratamento recebeu a mesma quantidade de líquido igualmente distribuída nas quatro repetições (correspondendo a 2501 de calda por hectare). Tal apli cação foi realizada de lado, a fim de possibilitar maiór eficiència dos produtos, sendo que não havia vento no campo. Utilizou-se o espallhante-adesivo "Extravon", em quant idade correspondente a $25 \mathrm{~cm}^{3} / 100$ I calda. Na apli cação adicionou-se em todos os tratamentos, incluida $\vec{a}$ testemunha, o endossulfano contra o bicudo do algodoeirc 


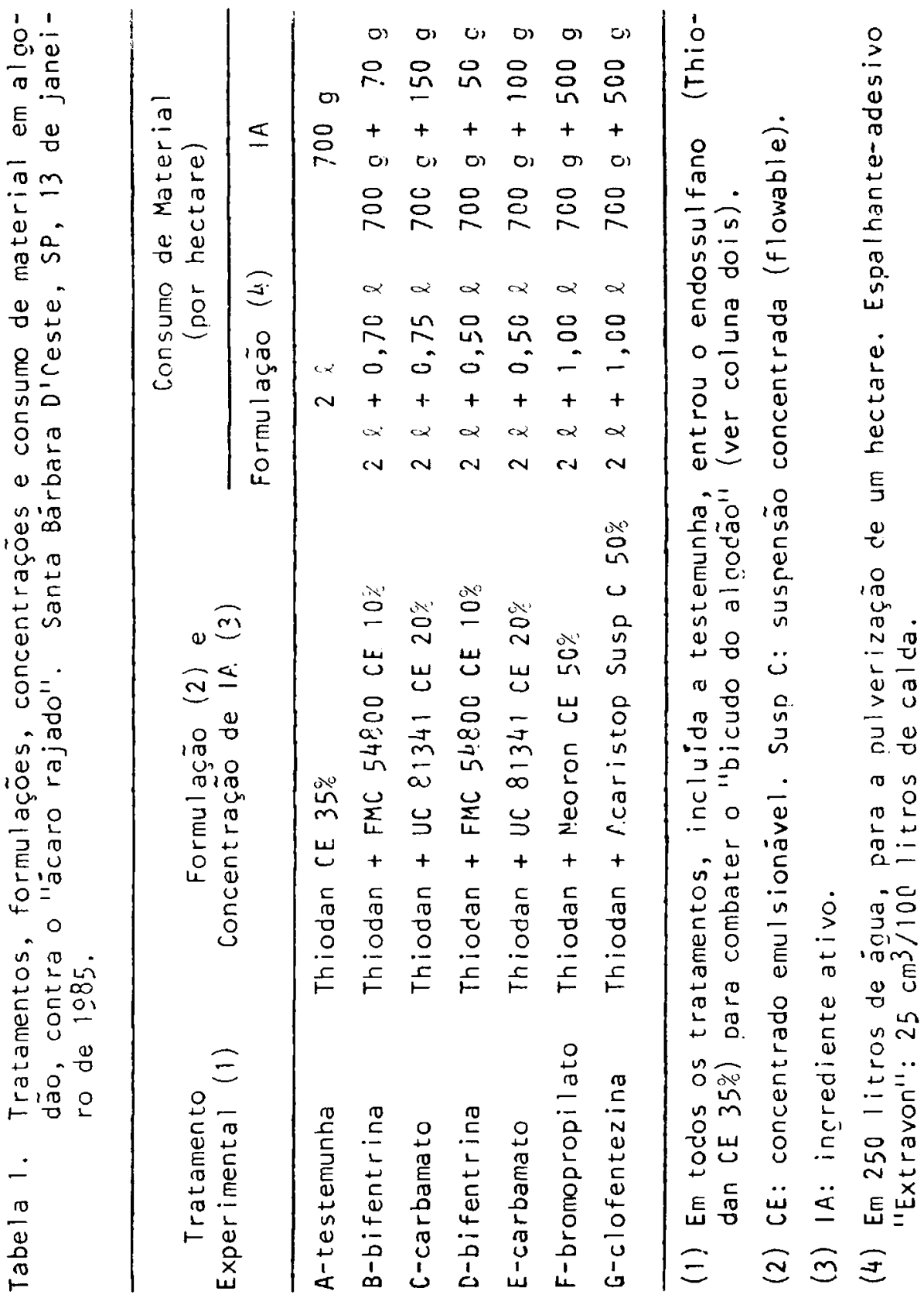


Anthonomus grandiz grandis. Posteriormente, realizaramse duas pulverizaçöes gerais no campo com endossulfano na mesina dosagem, feitas nos dias 29/01 e 07/02/85.

\section{Coletas}

Em cada avaliação coletaram-se 30 folhas por parce 1 a, sendo tomadas as que apresentassem sintomas de ataque do ácaro e correspondessem à 3 a ou 4a folha do terço superior da planta. Essas folhas eram colocadas em saquinhos plásticos rotulados e estes, acondicionados em caixa de isopor para proteção contra a radiação solar até que chegassem ao laboratōrio. Das nove linhas de al godão a que correspondia cada parcela, as sete linhas in ternas foram amostradas, deixando-se as duas linhas laterais como bordadura.

\section{Aval iações}

Realizaram-se sete avaliações em folhas (uma prévia e seis posteriores à aplicação). As datas e interva los foram 11/01/85 (prévia, 02 dias antes da aplicação), 15/01 (02 dias após a aplicação), 22/01 (09 dias apōs), 26/01 (13 dias após), 31/01 (18 dias após), 05/02 $(23$ dias apōs) e 11/02 (29 dias apōs).

\section{Contagens}

Realizadas em laboratōrio, utilizando-se lupas de mesa em aumento de 15 vezes, numa àrea circular de $15 \mathrm{~mm}$ de diāmetro, feita com vazador n: 10, na página in ferior das folhas, próximo ao pedúnculo e entre duas ne $\bar{r}$ vuras. Foram considerados apenas formas jovens e adul= tos vivos, não sendo contados os ovos do äcaro. Cada saquinho teve suas folhas separadas entre os värios auto res para as avaliações. As populaçōes de äcaros nó transcorrer do experimento podem ser vistas na Tabela 2. 


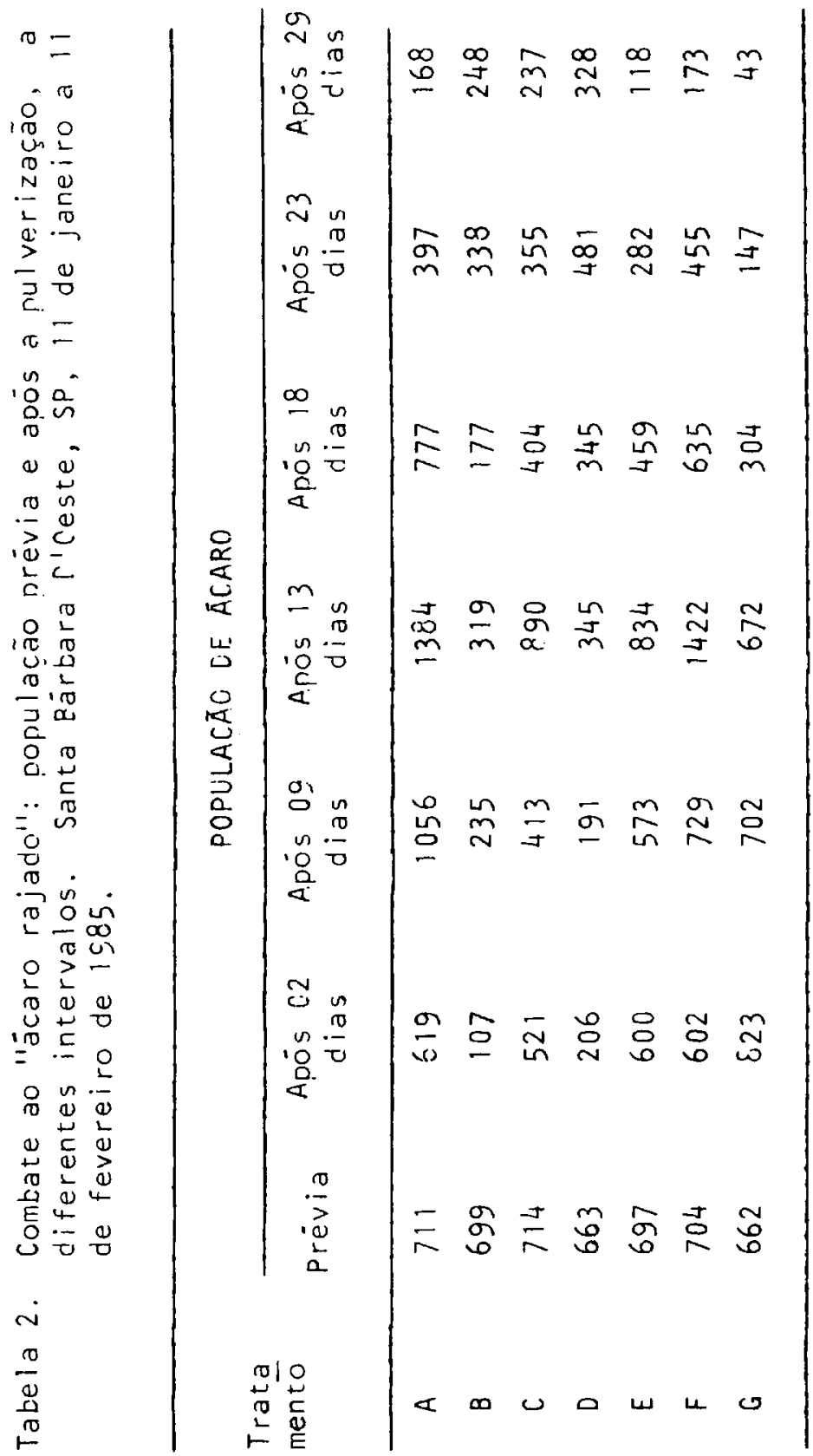




\section{Análise Estatísticas}

Os dados obtidos das contagens foram transformados em $\sqrt{x}$, sendo $x$ o número de ácaros de cada parcela, numa dada avaliação. Tais resultados foram submetidos à anä1 ise pelo teste " $F$ " e se apurada siçnificáncia, procedia - se à análise das médias pelo teste de "Tukey", ao nivel de 5\%. Foi estimado para cada avaliação o coeficiente de variaçāo, sendo que os resultados de tal análise podem ser vistos na Tabela 4.

\section{Redução Real}

Outro importante parámetro utilizado foi a eficièn cia ou mortalidade real (reduçāo real), calculado pelá fórmula transformada de Abbott, tomando-se por base a po pulação de ácaros de cada tratamento transformada em por centagem de sobrevivência. Tais dados encontram-se ná Tabela 3 .

\section{RESULTADOS E DISCUSSÃO}

Baseado na anālise estatística, a nivel de $5 \%$ de probabilidade, pode-se concluir os seguintes resultados: Contagem inicial: os tratamentos não diferem entre si; 1a contagem (02 dias): $B$ e $D$ (bifentrina 70 e $50 \mathrm{~g}$, respectivamente) diferem da testemunha; 2 . contagem (09 dias); os tratamentos B, C (carbamato UC $81341150 \mathrm{~g}$ ), D e E (carbamato UC $2134 \overline{1} 1 \overline{0} 0 \mathrm{~g}$ ) diferem da testemunha; $3 \overline{6}$ contagem (13 dias): os tratamentos $\underline{B}$, $\underline{D}$ e $G$ (clofentezi na $500 \mathrm{~g})$ diferem da testemunha, com as respectivas redu ções reais: $76,5 \%, 73,3 \%$ e $47,8 \%$; 4 ạ contagem (18 dias): os tratamentos B (bifentrina $70 \mathrm{~g}$ ), C (carbamato UC $81341150 \mathrm{~g}$ ), $\underline{D}^{-}$(bifentrina $50 \mathrm{~g}$ ) e $\mathrm{e}^{-}$(clofentezina $500 \mathrm{~g})$ diferem da testemunha. O tratameñto $\underline{B}$ destacou- 


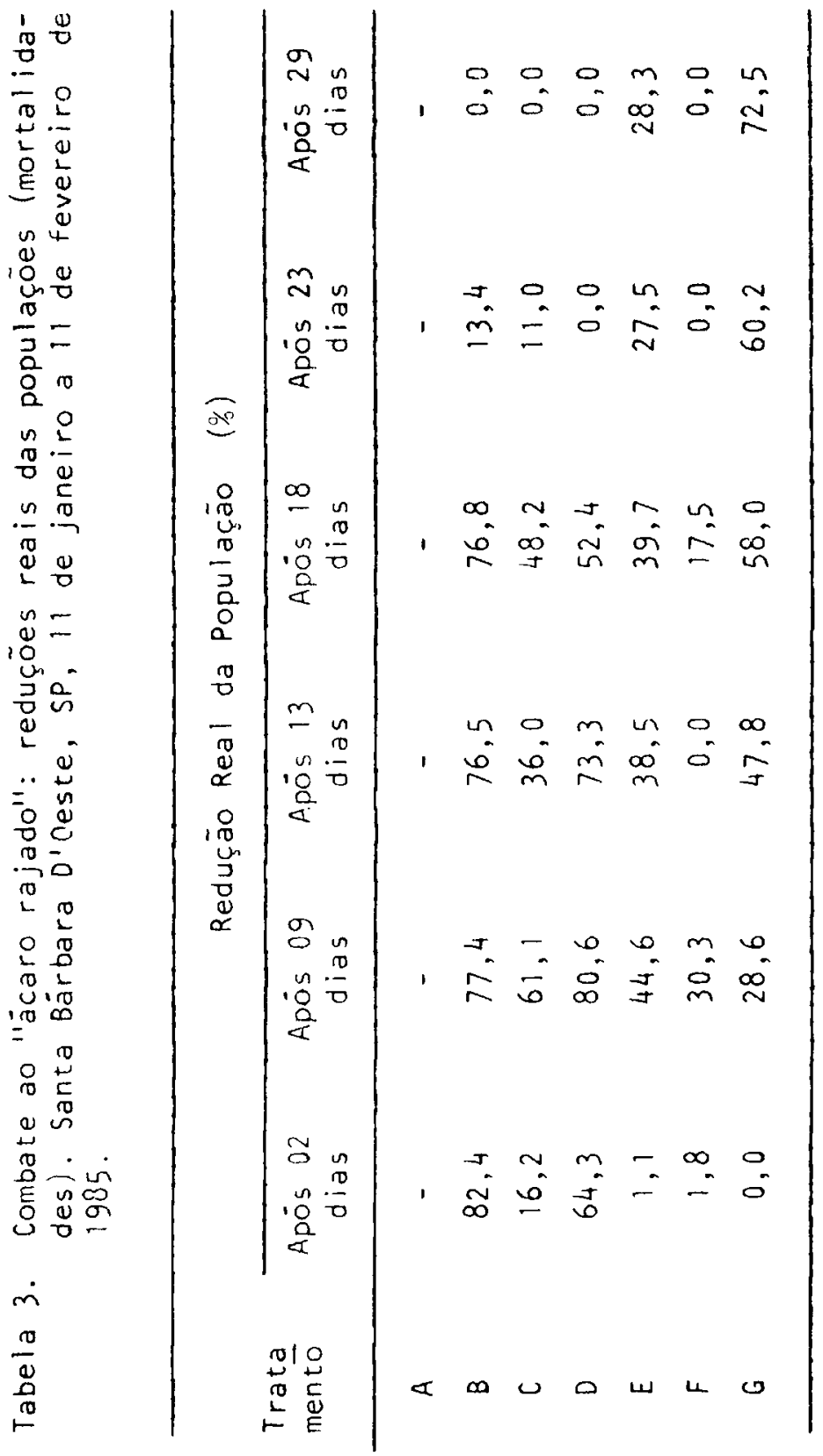




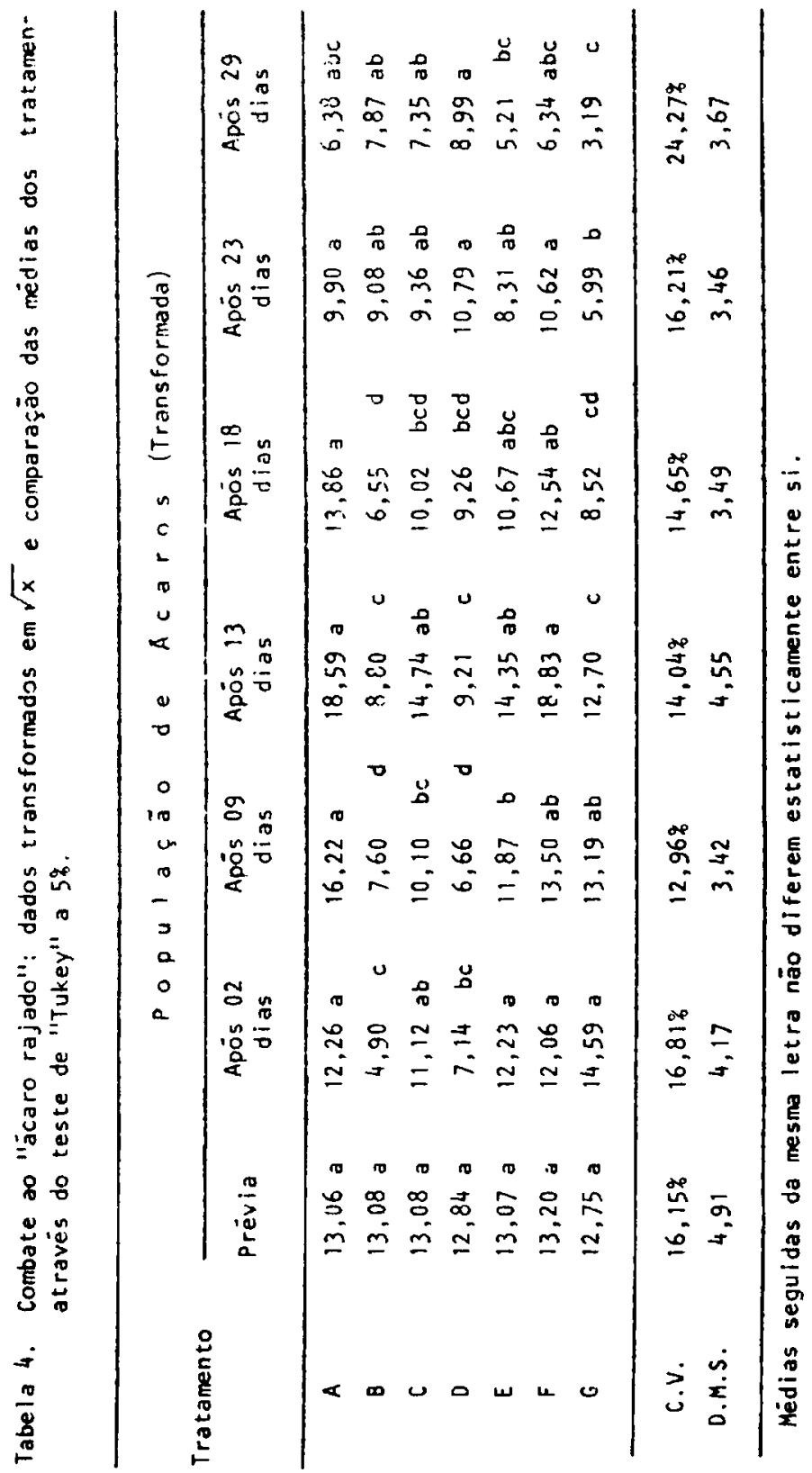


se entre os demais com uma redução real de $76,8 \%$; 5 a cor tagem $(23$ dias): apenas o tratamento $G$ diferiu da testemunha, apresentando uma eficiência de $60,2 \%$. o tratamento $B$ teve sua eficiência reduzida para $13,4 \%$; 6 a contagem (29 dias): nenhum dos tratamentos diferiu estatisticamente da testemunha. O tratamento G (clofentezina 500 g) apresentava uma eficiência de $72,5 \%$.

\section{CCNCLUSC̃ES}

Pela interpretação dos dados da anālise estatística e redução real, constatamos que a bifentrina na dosagem de $70 \mathrm{~g} / \mathrm{ha}$ mostrou-se muito eficiente até os $18 \mathrm{dias}$ após a aplicação. Além disso, verifica-se que a cló fentezina, na dosagem de $500 \mathrm{~g} / \mathrm{ha}$, constituiu excelente tratamento, apresentando ainda efeito residual relativamente longo.

Constatou-se uma demora na ação da clofentezina; isso deve-se ao fato do produto ser ovicida, atuando, portanto, preventivamente, contra as gerações futuras. Tal efeito explica porque ela não diferiu da testemunha no princípio.

SUMMARY

PESTICIDES AGFINST THE TWO-SPOTTED SPIDER

MITE T'etranyehus urtirat Koch, 1836 ON COTTON

This test was carried out in Santa Bärbara D'Ceste, State of São Paulo, Brazil.

The treatments and quantities of active ingre- 
dients per hectare vere: A - check (no pesticide); $B$ biphenthrin, $70 \mathrm{~g} ; C$ - carbamate UC $81341,150 \mathrm{~g}$; $\bar{D}$ biphenthrin, $50 \mathrm{~g}$; $\bar{E}$ - carbamate UC $\varepsilon 1341,100 \mathrm{~g}$; F-bromopropilate, $500 \mathrm{~g}$; $\mathrm{G}$ - clofentezine, $500 \mathrm{~g}$. Endosulfan was added to each experimental pesticide in order to con trol the cotton boll weevil Anthonoms arandis grandis Boh.

In the first avaluations the best control was obtained with biphenthrin. In the last avaluations, clofentezine was the best.

Key-words: the two-spotted spider mite, cotton, "ithangeths urtione, pesticides.

LITERATURA CITADA

ALMEIDA, S.L.; TAKAOKA, M.; TONIOLO, S.R.; KATO, W.Y.; PRANDINA, J. M.P.; SILVEIRA, I.; DOMINGUES, R. G.; BLANCO JR., R. E MARICONI, F.A.M., 1984. Pulverização de clofentezina, fempropatrina e firetrōide "FMC $54 \varepsilon 00$ " contra o "äcaro rajado" tetrarizrihus ur.ticele: Koch, $1 \varepsilon .36$, em plantação de al godão. O Solo, Piracica ba, São Paulo, $76(2): 29-33$.

ALVES, A.D. E SALGADC, L.0., 1984. Controle químico do äcaro rajado Tetranys hus urticue Koch, 1836 (AcariTetranychidae) em algodoe iro tom MK 936 1,8\% EC. In: IX Congresso Brasileiro de Entomologia, Londrina, Paranā. Resumos, p. 232.

KATO, W.Y.; TONIOLO, S.R.; SILVEIRA, I.; BLANCO JR., R.; DOMINGUES, R.G.; TAKAOKA, M.; ALMEIDA, S.L. \& MARICONI, F.A.M., 1984. Eficiéncias de inseticidas no combate ao "ácaro rajado" T'etranychus urticue Koch, 1836, em cultura de algodão. In: Anais do IV Congres- 
so Brasileiro de Iniciação Científica em Ciências Agrärias, Taubaté, S. Paulo, p. 142.

MARICCNI, F.A.M.; F.LMEIOA, S.L.; GF.LHARDO, L.C.S.; COR TE, C.R.; MORAIS, A.A.; SOARES, R.L.; GODOY, J.M.T. $\bar{\varnothing}$ MORAES, L.F.D., 1983. Pulverizaçĩic experimental contra o äcaro rajado Tetranychus urticae Koch, $1836 \mathrm{em}$ al codoeiro. In: VIII Congresso Brasileiro de Entomologia, Brasillia, DF. Resumos, p. 106.

MORF.IS, A.P..; CORTE, C.R.; FEKETE, T.J.; ALMEIDA, S.L.; GALHARDO, L.C.S. \& MARICONI, F.A.M., 1981. Pulveriza ções em algodão contra Tetranychus urticae Koch, 1836 "äcaro rajado". In: VII Congresso Brasileiro de Entomologia, Fortaleza, Ceará. Resumos, p. T. 184.

OLIVEIRA, C.A.L., 1971. Estudo dos prejuizos quantitati vos e qualitativos determinados pela infestação do ácaro rajado Tetranychus urticue Koch, 1836, na cultu ra de algodão. O Biológico, S. Paulo $37(12)$ : $341=$ 342 .

PEDRCNI, D.; SILVEIRA, D.F.; SMITH, R.K. E TENCATTI, J., 1984. O pulverizador Electrodyn em algodoeiro: controle das pragas Anthonomus grandis, Tetranychus urt $i$ cae e Polyphagotarsonems Latus. In: Ix Congresso Brä sileiro de Entomolocia, Londrina, Paraná. Resumos p. 222.

PEREZ, C.A.; ROSA, N.L.V. E NAKANO, 0., 1970. Controle químico do "àcaro rajado" Tetranychus (T.) urticae, através de pulverizaçöes diretas e indiretas em cultu ra de algodão. In: V Congresso Brasileiro de Entomologia, Il héus-ltabuna, Bahia. Resumos p. s/n?.

SANTOS, M.S.T.A.; PEDREIRO JR., R.; P.LMEIDA, S.L.; GODOY, J.M.T.; CORTE, C.R.; QUEIROZ, C.A.G. E MA.RICONI, F.A. M., 1983. Defensivos quImicos pulverizados em algodäo contra o "äcaro rajado" Tetranychus urticae Korh, 1836. In: Anais do 111 Congresso Brasileiro de Ini- 
ciação Científica em Cièncias Agrárias, florianópolis, S. Catarina, p. 26.

SANTOS, W.J., 1984. Avaliação do controle do ácaro raja do Tetranychua urticae Koch, 1836 em algodoeiro, In: IX Congresso Brasilelro de Entomologia, Londrina, Paranā. Resumos, p. 233.

TA.KF:OKA, M.; DOMINGUES, R.G.; PRANDINA, J.M.P.; KATO, $\because$. Y.; TONIOLO, S.R.; ALMEIDA, S.L.; SILVEIRA, I.; BLANCO JR., R. \& MARICCNI, F.A.M., 1984. Pulverização de defensivos simples e misturas contra o "ácaro rajado" Tetranychus urticae Koch, 1836 em algodäo. In: IX Con gresso Brasileiro de Entomologia, Londrina, Paraná. Resumos, p. 235. 